\title{
Gastrointestinal Stromal Tumor Mimicking Arteriovenous Malformation of the Jejunum
}

\author{
Kazue Shiozawa ${ }^{a}$ Manabu Watanabe $^{a}$ Yoshinori Igarashia $^{a}$ \\ Mioe Ichimori ${ }^{a}$ Naoki Hirano ${ }^{a}$ Shigeru Nakano ${ }^{a}$ \\ Tetsuya Maeda $^{\text {b }}$ Kunihiro Yamazaki ${ }^{\text {b }}$ Youichiro Okubo ${ }^{c}$ \\ Tetsuo Nemoto $^{c}$ Kazutoshi Shibuya ${ }^{c}$ Yasukiyo Sumino ${ }^{a}$ \\ Departments of a Hepatology and Gastroenterology, ${ }^{\mathrm{b} S}$ Surgery and 'Surgical \\ Pathology, Toho University Medical Center, Omori Hospital, Tokyo, Japan
}

\section{Key Words}

Gastrointestinal stromal tumor - Arteriovenous malformation · Contrast-enhanced computed tomography - Contrast-enhanced ultrasonography

\begin{abstract}
There have been case reports of small intestinal gastrointestinal stromal tumors (GISTs) complicated with arteriovenous malformation (AVM) and angiodysplasia and exhibiting intense tumor staining. Herein we report a GIST of the small intestine that showed tumor staining and early venous return on imaging studies, and so the patient was suspected to have AVM. A 62-year-old male presented with intermittent pain in the left abdominal region. Contrast-enhanced computed tomography revealed a 15 -mm-long spindle-shaped mass showing intense tumor staining and early venous return through the jejunal vein. In the arterial phase, the attenuation value of the tumor was 250 Hounsfield units. Color Doppler ultrasonography simultaneously delineated vessels extending from the serosal side and turbulent signals showing a mosaic pattern in the tumor. On angiography, intense staining was observed in the peripheral part of the second branch of the jejunal artery. Although these findings suggested AVM, the tumor was diagnosed as a GIST based on pathological examination of the resected specimens. In this case, no AVM or change in vascular density was noted despite the careful examination of pathological specimens, and the cause of the tumor staining remained unknown.
\end{abstract}




\section{Introduction}

Gastrointestinal stromal tumors (GISTs) are mesenchymal neoplasms affecting the gastrointestinal tract and consisting of CD34-positive and c-Kit signaling-driven cells [1]. Up to the present, tumors arising in the muscle layer of the gastrointestinal tract have generally been considered to originate from smooth muscle cells, and GISTs have also been diagnosed as leiomyomas, leiomyoblastomas, or leiomyosarcomas. However, since Hirota et al. [2] reported the expression of Kit and an activating mutations of the c-Kit gene in GISTs in 1998, the concept that GISTs are special tumors of the gastrointestinal tract originating from the interstitial cells of Cajal has been established.

By imaging studies, GISTs are delineated generally as well-defined tumors showing homogeneous contrast enhancement on computed tomography (CT) [3] and often as markedly hypoechoic, well-demarcated round masses by ultrasonography (US) [4]. However, these findings may vary with the malignant potential of tumors [5].

We describe a patient who presented with left abdominal pain as the primary complaint, showed tumor staining and early venous return on imaging studies, and so was suspected to have arteriovenous malformation (AVM), but was diagnosed with GIST of the small intestine based on pathological examination of the resected specimens. Cases of small intestinal GISTs complicated with AVM and angiodysplasia and exhibiting intense tumor staining have been reported $[6,7]$. In this case, no AVM or change in vascular density was noted despite careful examination of the pathological specimens, and the cause of the tumor staining remained unknown. Since this case is thought-provoking, it is reported with a review of the literature.

\section{Case Report}

The patient was a 62-year-old male orally treated with thyradin for hypothyroidism. He developed intermittent pain in the left abdominal region around March 2010, and as he had experienced several episodes of intense abdominal pain accompanied by cold sweats over about 1 month, he was admitted to our hospital. On admission, initial physical examination revealed a flat and soft abdomen and mild tenderness in the left abdominal region. Blood tests suggested no abnormality such as increased inflammatory reaction or anemia.

Contrast-enhanced CT (CECT) using a contrast medium containing $300 \mathrm{mg}$ of iodine (Iomeron 300 , Eisai, Tokyo, Japan) at a flow rate of $3 \mathrm{ml} / \mathrm{s}$ revealed a $15-\mathrm{mm}$-long spindle-shaped mass showing intense tumor staining and early venous return through the jejunal vein, which was continuous with the mass. In the arterial phase, the attenuation value of the tumor was 250 Hounsfield units (HU), and weak staining persisted in the equilibrium phase (ig. 1a). On US, a low-echoic mass $14 \mathrm{~mm}$ in diameter with a clear margin not accompanied by posterior acoustic enhancement was observed in the jejunal wall. Color Doppler US simultaneously delineated vessels extending from the serosal side and turbulent signals showing a mosaic pattern in the mass. No wall thickening was noted in the intestine adjacent to the mass (fig. 1b). We performed contrast-enhanced US (CEUS) using Sonazoid (Diichi-Sankyo, Tokyo, Japan) and observed homogeneous staining in the mass in the early vascular phase and persistence of staining in the late vascular phase. No defect was noted inside the mass during the examination. Capsule endoscopy showed no clear abnormality in the small intestine, even in the area suspected to be the site of the lesion in the jejunum. On angiography, intense staining was observed in the peripheral part of the second branch of the jejunal artery. Angiographic CT delineated a large jejunal vein continuous with the mass nearly simultaneously with injection of the contrast medium (fig. 1c).

Although these findings suggested AVM, a tumorous lesion such as GIST and carcinoid could not be excluded, and laparoscopic intestinal resection was carried out. A mass about $15 \mathrm{~mm}$ in diameter was palpated in the mesojejunum about $20 \mathrm{~cm}$ from the ligament of Treitz, and partial jejunectomy 
including this mass was performed. On gross examination of the resected sample, the mass measured $14 \times 9 \mathrm{~mm}$ ( $\underline{\text { fig. }}$. $\mathrm{a}$ ), and its cross-section was grayish white and solid. Histopathologically, tumor cells proliferated from the lower mucosal to the lower serosal layer. The tumor cells had a relatively short elliptical shape, a large, hyperchromatic and somewhat irregularly shaped nucleus, and spindle-shaped cytoplasm, and grew in a vortical pattern (fig. 2b). Since c-Kit was positive on immunostaining, the tumor was histopathologically diagnosed as GIST. The mitotic count was $<5 / 50$ high-power fields (HPFs).

\section{Discussion}

GISTs are relatively rare tumors but are the most common mesenchymal tumors of the gastrointestinal tract. Rosai [8] classified GIST into four categories: (1) a smooth muscle type positive for myogenic markers such as smooth muscle actin and desmin, (2) a neural type positive for neurogenic markers such as S-100 proteins and neuron-specific enolase, (3) a combined smooth muscle-neural type exhibiting properties of both the smooth muscle and neural types, and (4) an uncommitted type with no tendency to differentiate into any particular type and positive for CD34, a marker of juvenile mesenchymal cells such as vascular endothelial cells and undifferentiated bone marrow hematopoietic stem cells, and c-Kit, a receptor type tyrosine kinase. The majority of tumors occur in the stomach $(60-70 \%)$ and small intestine $(25-35 \%)$ with rare occurrences in the colon and rectum (5\%), esophagus $(<2 \%)$, and appendix [9]. Symptoms are unlikely to appear until the tumor grows to a certain size, but the most common symptom is gastrointestinal bleeding and occasionally also abdominal pain and bowel obstruction. Histopathological examination is indispensable for a definitive diagnosis, but CT is considered useful for the detection and evaluation of GISTs [10]. Endoscopic ultrasonography is useful as well for the assessment of submucosal masses located within the reach of an endoscope [11]. There are also reports primarily regarding US and CEUS findings $[5,12,13]$.

Generally, tumor size, mitotic count and presence or absence of invasion or metastasis are used to define the risk of aggressive behavior in GISTs [1], and those with a tumor size $>5 \mathrm{~cm}$ and a mitotic count $>5 / 50 \mathrm{HPFs}$, those with a tumor size $>10 \mathrm{~cm}$ regardless of mitotic count, and those with a mitotic count $>10 / 50$ HPFs regardless of tumor size are considered a high-risk group [14]. On US, small GISTs have relatively well-demarcated round and homogeneous internal echo structure accompanied by posterior acoustic enhancement. Large GISTs often present as solid masses with more heterogeneous internal echo structure and contain intratumoral hypoechoic areas suggesting hemorrhage or necrosis [4]. The high malignant potential on US is the internal heterogenicity with the presence of intratumoral hypoechoic areas [5]. In this case, tumor size was slightly smaller than $15 \mathrm{~mm}$, and the mitotic count was $<5 / 50 \mathrm{HPFs}$, indicating a very-low-risk group, and the tumor appeared as a clearly bordered hypoechoic mass on US.

Color Doppler US imaging usually shows blood flow signals in the internal tumor, and the blood flow varies from homogeneous to heterogeneous. In this case, turbulent signals showing a mosaic pattern were noted in the tumor, but to date there has been no report of GISTs of the small intestine showing turbulent signals. Fukuta et al. [12], on the other hand, evaluated the vascularity and malignant potential of GISTs by CEUS using Levobist (Schering AG, Berlin, Germany). The CEUS images were classified into two types according to the blood flow area of the tumors as seen by real-time continuous imaging of the tumor vessels, and the authors reported that those with poor vascularity were likely to 
be benign but those with rich vascularity to be malignant, and that the vascularity on CEUS was correlated with tissue vascular density. In this case, CEUS using Sonazoid showed homogeneous enhancement in the vascular phase, and the tumor had a rich vascularity but was benign, unlike in the report by Fukuta et al. This was probably because the 13 patients reported by them had relatively large tumors of 20-120 mm (median $43.5 \mathrm{~mm}$ ) in diameter.

$\mathrm{CT}$ is used for the evaluation of invasion pattern, stage and therapeutic effects and is useful for the examination of GISTs in the small intestine or more distal parts, which can be difficult to visualize by endoscopic ultrasonography [9]. On CECT, GISTs are normally seen as well-defined, round or oval, exophytic masses that show soft tissue attenuation and homogeneous enhancement [3]. Lee et al. [15] compared the plain and equilibrium phases in CT evaluation of 17 GIST patients, and reported that the post-contrast attenuation of the tumor was $70 \%$ higher than the pre-contrast one. However, there has been no report comparing the attenuation values of pre-contrast with those of post-contrast in the arterial phase. In this case, the attenuation value was determined in the arterial phase $35 \mathrm{~s}$ after the administration of $90 \mathrm{ml}$ of a contrast medium by setting a region of interest over the entire tumor. The attenuation value of the tumor was $250 \mathrm{HU}$, the attenuation value of the abdominal aorta in the same phase was $290 \mathrm{HU}$. When the attenuation values in the arterial phase were calculated in 3 GIST patients with a tumor size $\leq 25 \mathrm{~mm}$ (mean $23.7 \pm 1.5 \mathrm{~mm}$ ) who underwent CECT at our facility during the 3 years from 2008 to 2010 under the same conditions including the timing of contrast enhancement and the amount of the contrast medium, the mean attenuation value of these tumors was $139.7 \pm 29 \mathrm{HU}$, and none showed early venous return. The attenuation value of our case was markedly higher than the mean of these 3 patients, indicating that the tumor of our case had a significantly richer vascularity (fig. 3 ).

In conclusion, there have been reports of GISTs which had complications such as AVM and angiodysplasia and showed intense tumor staining $[6,7]$. Since this case simultaneously exhibited intense tumor staining on CECT and return through an enlarged jejunal vein, we initially suspected AVM, but no sign of AVM was noted on close examination of the pathological specimens, and the cause of the intense tumor staining in this patient remained unclear. Its cause may be clarified in the future by comparatively and histopathologically evaluating the number of vessels and vascular density in small GISTs similar to our case. 

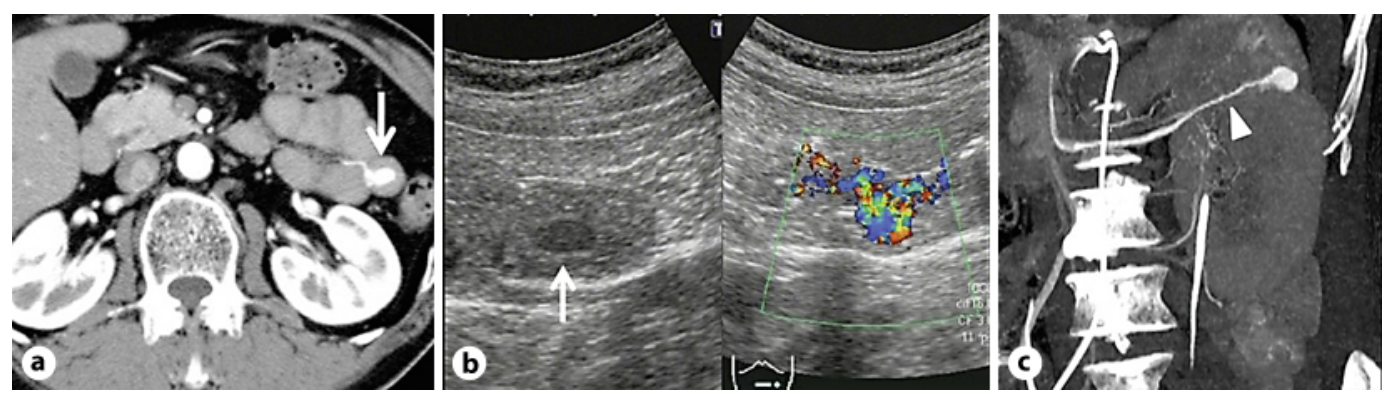

Fig. 1. a CECT revealed a long spindle-shaped mass showing intense tumor staining and early venous return through the jejunal vein, which was continuous with the mass (arrow) in the early phase. b Horizontal US scan revealed a low-echoic mass (arrow) with a clear margin in the jejunal wall. Horizontal color Doppler US scan simultaneously delineated vessels extending from the serosal side and turbulent signals showing a mosaic pattern in the mass. c Angiographic CT delineated a large jejunal vein (arrowhead) continuous with the mass nearly simultaneously with injection of the contrast medium.
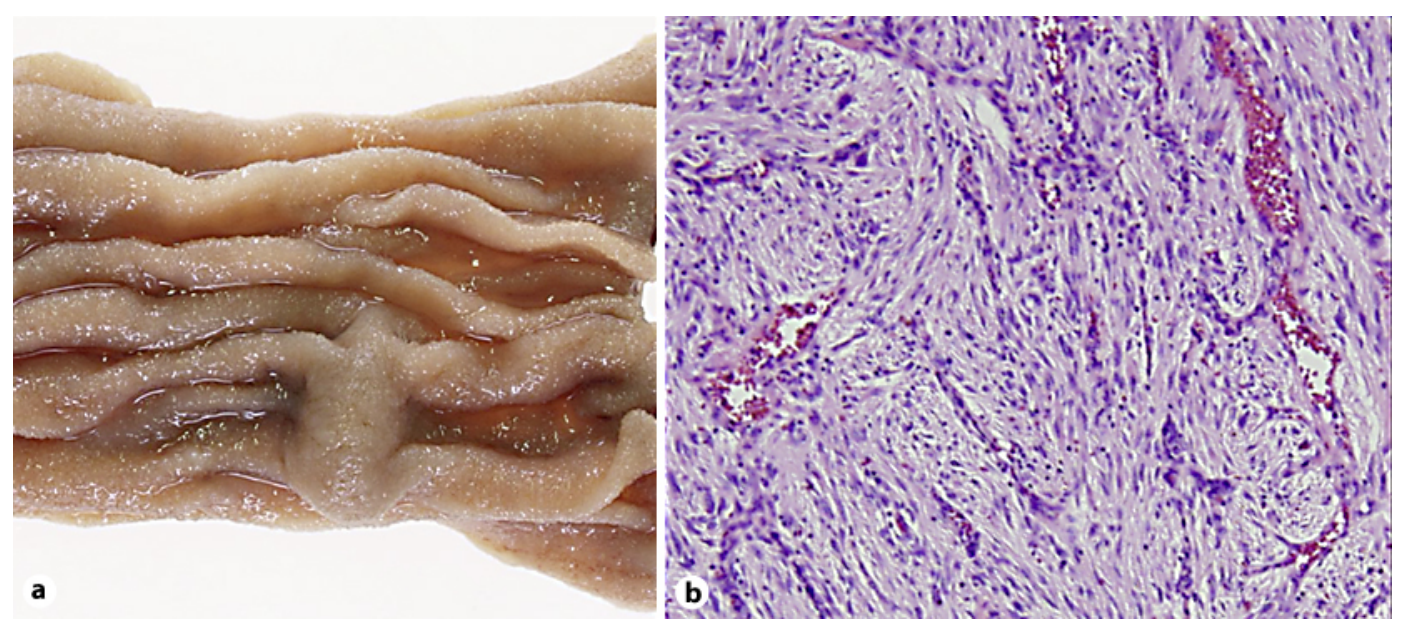

Fig. 2. a On gross examination of the resected sample, the mass measured $14 \times 9 \mathrm{~mm}$ in the mucosal side. b Histopathologically, the tumor cells had a relatively short elliptical shape, a large, hyperchromatic and somewhat irregularly shaped nucleus, and spindle-shaped cytoplasm (HE stain, $\times 100)$. 


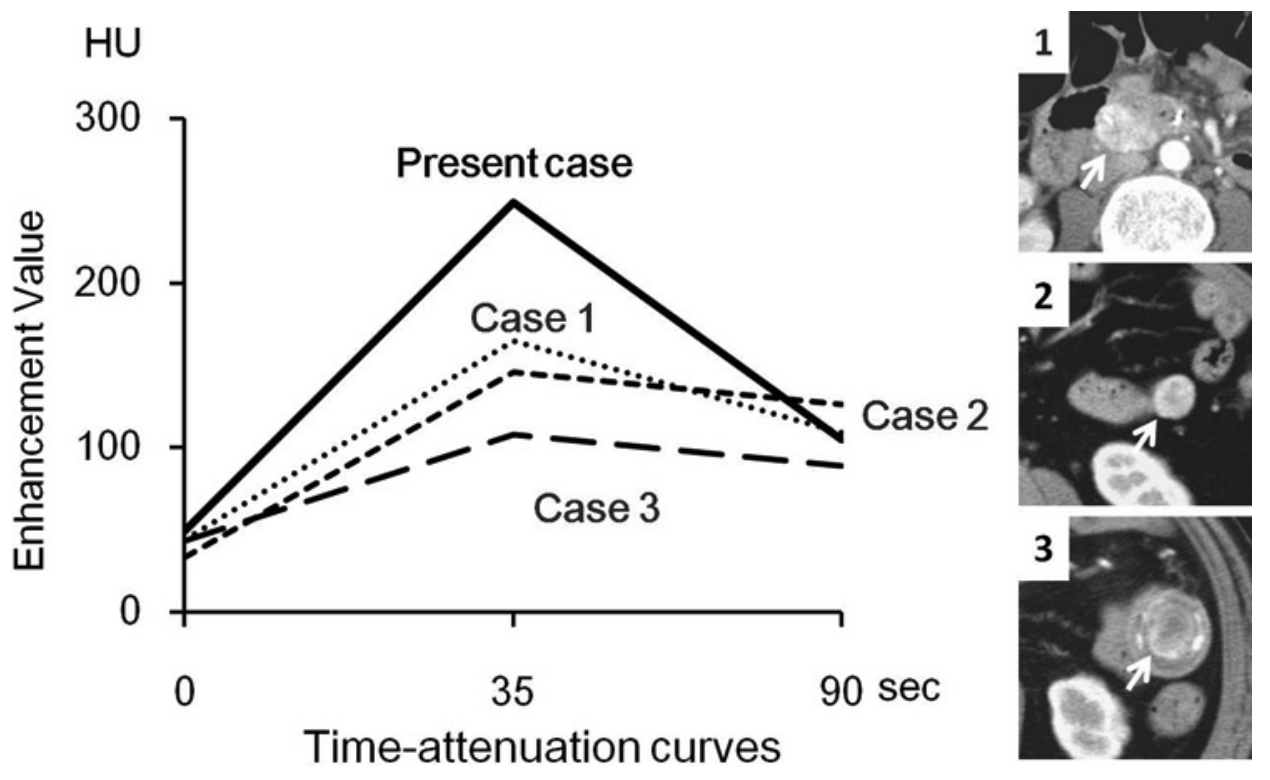

Fig. 3. Time-attenuation curves of small intestinal GISTs $<25 \mathrm{~mm}$ in size previously experienced in 3 cases (arrows). In the arterial phase $35 \mathrm{~s}$ after the contrast medium, the enhancement value of the tumor in our case showed higher than the enhancement values of the tumors in cases 1-3.

\section{References}

1 Darnell A, Dalmau E, Pericay C, Musulén E, Martín J, Puig J, Malet A, Saigí E, Rey M: Gastrointestinal stromal tumors. Abdom Imaging 2006;31:387-399.

$\checkmark 2$ Hirota S, Isozaki K, Moriyama Y, Hashimoto K, Nishida T, Ishiguro S, Kawano K, Hanada M, Kurata A, Takeda M, Muhammad Tunio G, Matsuzawa Y, Kanakura Y, Shinomura Y, Kitamura Y: Gain-of-function mutations of c-kit in human gastrointestinal stromal tumors. Science 1998;279:577-580.

-3 Ghanem N, Altehoefer C, Furtwängler A, Winterer J, Schäfer O, Springer O, Kotter E, Langer M: Computed tomography in gastrointestinal stromal tumors. Eur Radiol 2003;13:1669-1678.

4 Beggs I: Sonographic appearances of nerve tumors. J Clin Ultrasound 1999;27:363-368.

5 Wronski M, Cebulski W, Slodkowski M, Krasnodebski IW: Gastrointestinal stromal tumors: ultrasonographic spectrum of the disease. J Ultrasound Med 2009;28:941-948.

-6 Shim SH, Han YH, Kwak JE, Chang SH, Kim H, Chi JG, Joo M: Synchronous development of gastrointestinal stromal tumor and arteriovenous malformation in the jejunum. A case report. Korean J Pathol 2008;42: $185-188$.

7 Tomita T, Palacherla J, Zuckerman M, Dougherty S, Ghaleb M: Gastrointestinal stromal tumor of jejunum with angiodysplasia. Dig Dis Sci 2004;49:667-671.

8 Rosai J: Stromal tumors; in Rosai J (ed): Ackerman's Surgical Pathology, ed 8. St. Louis, Mosby-Year Book, 1996, pp 645-647.

-9 Miettinen M, Lasota J: Gastrointestinal stromal tumors - definition, clinical, histological, immunohistochemical, and molecular genetic features and differential diagnosis. Virchows Arch 2001;438: $1-12$.

10 Lau S, Tam KF, Kam CK, Lui CY, Siu CW, Lam HS, Mak KL: Imaging of gastrointestinal stromal tumour (GIST). Clin Radiol 2004;59:487-498.

-11 Akahoshi K, Sumida Y, Matsui N, Oya M, Akinaga R, Kubokawa M, Motomura Y, Honda K, Watanabe M, Nagaie T: Preoperative diagnosis of gastrointestinal stromal tumor by endoscopic ultrasound-guided fine needle aspiration. World J Gastroenterol 2007;13:2077-2082. 
12 Fukuta N, Kitano M, Maekawa K, Chikugo T, Kudo M: Estimation of the malignant potential of gastrointestinal stromal tumors: the value of contrast-enhanced coded phase-inversion harmonics US. J Gastroenterol 2005;40:247-255.

13 Girtler MT, De Zordo T, Romagnoli C: Sonographic findings in a patient with neurofibromatosis type 1 and a gastrointestinal stromal tumor. J Clin Ultrasound 2010;38:274-278.

-14 Fletcher CD, Berman JJ, Corless C, Gorstein F, Lasota J, Longley BJ, Miettinen M, O’Leary TJ, Remotti H, Rubin BP, Shmookler B, Sobin LH, Weiss SW: Diagnosis of gastrointestinal stromal tumors: a consensus approach. Hum Pathol 2002;33:459-465.

15 Lee CM, Chen HC, Leung TK, Chen YY: Gastrointestinal stromal tumor: computed tomographic features. World J Gastroenterol 2004;10:2417-2418. 